\title{
Quality of life of patients with nasal bone fracture after closed reduction
}

\author{
Young Ji Park, \\ Gi Cheol Do, \\ Gyu Hyeon Kwon, \\ Woo Sang Ryu, \\ Kyung Suk Lee, \\ Nam Gyun Kim
}

Department of Plastic and Reconstructive Surgery, Institute of Health Sciences, Gyeongsang National University Hospital, Gyeongsang National University School of Medicine, Jinju, Korea

\begin{abstract}
Background: Closed reduction is the standard treatment for nasal bone fractures, which are the most common type of facial bone fractures. We investigated the effect of closed reduction on quality of life.

Methods: The 15-dimensional health-related quality of life survey was administered to 120 patients who underwent closed reduction under general anesthesia for nasal bone fractures from February 2018 to December 2019, on both the day after surgery and 3 months after surgery. Three months postoperatively, the presence or absence of five nasal symptoms (nose obstruction, snoring, pain, nasal secretions, and aesthetic dissatisfaction) was also evaluated.

Results: The quality of life items that showed significant changes between immediately after surgery and 3 months postoperatively were breathing, sleeping, speech, excretion, and discomfort. Low scores were found at 3 months for breathing, sleeping, and distress. There were 31 patients $(25.83 \%)$ with nose obstruction, $25(20.83 \%)$ with snoring, $12(10.00 \%)$, with pain, 11 $(9.17 \%)$ with nasal secretions, and $29(24.17 \%)$ with aesthetic dissatisfaction.

Conclusion: Closed reduction affected patients' quality of life, although most aspects improved significantly after 3 months. However, it was not possible to rule out deterioration of quality of life due to complications and dissatisfaction after surgery.
\end{abstract}

Keywords: Facial bone / Nasal bone / Quality of life / Reduction

\section{INTRODUCTION}

Nasal bone fractures, which are the most common type of facial bone fractures [1-5], often result in structural or functional complications. Surgical results vary depending on the type of nasal bone fracture and/or the presence of septal fracture or deviation, even after surgery. Surgical treatments for nasal bone fracture include closed reduction, open reduction, and rhinoplasty, of which closed reduction is the standard treatment because it enables safe, simple, and effective reduction $[1,2]$. Previous studies have dealt with aesthetic and structural aspects of

\section{Correspondence: Nam Gyun Kim}

Department of Plastic and Reconstructive Surgery, Gyeongsang National

University Hospital, 79 Gangnam-ro, Jinju 52727, Korea

E-mail: pellow@hanmail.net

Received September 9, 2020 / Revised October 9, 2020 / Accepted October 20, 2020 nasal bone reduction and postoperative patient satisfaction limited to the nasal bone [2,3,6]. Choi et al. [2] studied differences in aesthetic and functional satisfaction with closed reduction according to the fracture type, site, and severity. Kang and Han [3] and Park et al. [6] measured objective outcomes through facial computed tomography. However, little is known about how facial bone fracture reduction affects health-related quality of life, with the exception of the study conducted by Kaukola et al. [7] on patients with zygomatic fractures. Therefore, this study evaluated changes in overall quality of life and its dimensions in patients who underwent closed nasal fracture reduction not specifically associated with nose function. The 15-dimensional health-related quality of life (15D-HRQoL) survey [8] was administered immediately after surgery and at a long-term ( 3 months) follow-up. 


\section{METHODS}

\section{Patients}

A total of 120 patients aged 18 and over who were diagnosed with acute nasal bone fracture from February 2018 to December 2019 completed the survey. Patients with open fracture, other facial bone fractures (e.g., zygoma fracture, frontal sinus fracture, or orbital wall fracture), and a previous history of nasal bone fracture or nasal surgery (e.g., rhinoplasty or septoplasty) were excluded. Only patients with complete bilateral fractures who underwent nasal packing after bilateral bone reduction were included.

\section{Surgical procedure}

In all patients, operations were performed by the same surgeon (NGK) using closed reduction under general anesthesia. In order to anesthetize the local area and reduce the risk of bleeding, a gauze containing $1 \%$ lidocaine and 1:100,000 epinephrine was packed into the nasal mucosa for about 10 minutes. The fracture site was confirmed by physical examination and orbital three-dimensional computed tomography in advance, and then denoted with a marker. After manual palpation inside the nasal cavity and checking the fracture site, the elevator was positioned and lifted in the lateral or superior direction. The symmetry and continuity of the nasal bone were confirmed by visual inspection and palpation. After reduction, Merocel (Fabco Inc., London, UK) was packed directly below the fractured area in the nostrils on both sides, and external splints (Megan Inc., Seoul, Korea) were positioned outside the nose to maintain and protect the nasal bone contour.

\section{D-HRQoL questionnaire}

The $15 \mathrm{D}-\mathrm{HRQ}$ oL is a generic, comprehensive, 15-dimensional, standardized, self-administered measure of health-related quality of life (HRQoL) that can be used both as a profile and single index score measure [8]. The 15 dimensions measured on the 15D-HRQoL are mobility, vision, hearing, breathing, sleeping, eating, speech, excretion, usual activities, mental function, discomfort and symptoms, depression, distress, vitality, and sexual activity.

The meaning of each item is as follows: (1) mobility refers to whether one can walk indoors or outdoors; (2) vision refers whether one can read the letters in a newspaper or on TV well; (3) hearing refers to the ability to hear and speak well without hearing aids; (4) breathing deals with whether there is any difficulty in breathing due to changes in body movements; (5) sleeping refers to whether one can sleep normally without the help of medication or a break in the middle; (6) the eating di- mension evaluates whether one can eat by oneself; (7) speech refers to whether one can speak fluently without stuttering; (8) excretion refers to whether there are problems with urination and defecation; (9) usual activities correspond to whether one can easily perform work, housework, studying, and other routine activities; (10) mental function indicates whether one can think logically without memory problems; (11) discomfort and symptoms deal with whether there is any physical discomfort such as pain, itchiness, or nausea; (12) depression refers to whether one is depressed or melancholic; (13) distress refers to whether one experiences nervousness, tension, or anxiety; (14) vitality refers to whether one feels healthy or energetic; and (15) sexual activity deals with whether one's current health conditions affect sexual life.

Responses for each dimension were categorized into five nonoverlapping levels that ranged from no problems to severe problems. Respondents chose the level that best described their current health status by ticking the appropriate box. The 15DHRQoL score represents overall HRQoL and ranges from a maximum score of 1 (full health) to a minimum score of 0 (equivalent to being dead) [9].

The 15D-HRQoL questionnaire was given to patients the day after surgery during hospitalization, while the 3-month followup surveys were conducted at our outpatient clinic. At the 3-month follow-up, five other questions were asked about the functional and cosmetic aspects of the nose, focusing on the presence or absence of nasal symptoms and dissatisfaction or satisfaction.

\section{Statistical analysis}

In the statistical comparison of 15D-HRQoL scores between the day after surgery and the 3-month follow-up, the data for all 15 dimensions did not satisfy the normality assumption, so the paired $t$-test (a parametric test) was not performed. Instead, the $p$-value was derived by performing the Wilcoxon signed-rank test (a nonparametric test). In addition, the results for both time points were expressed as a bar graph using average values and 95\% confidence intervals. The demographic characteristics of patients were expressed as percentages (e.g., for sex and history of injuries) or as mean and standard deviation (for age). The statistical program R 3.6.3. (R Core Team, Vienna, Austria, 2019) was used for all items.

\section{RESULTS}

Of the 120 patients, 92 were men and 28 were women, and the average age was 36.1 years. The most common causes of trauma were falling or slipping $(n=36)$, followed by traffic accidents 
$(n=24)$, assault $(n=20)$, sports-related accidents $(n=20)$, and other causes $(n=20)$. Other causes included injuries due to fly-

Table 1. Demographics of the patients included in this study

\begin{tabular}{lc}
\hline Characteristics & Value \\
\hline Sex & \\
Male & $92(76.67)$ \\
Female & $28(23.33)$ \\
Age (yr) & $36.10 \pm 17.73$ \\
Main causes of nasal bone fracture & \\
Assault & $20(16.67)$ \\
Traffic accident & $24(20.00)$ \\
Falling or slipping & $36(30.00)$ \\
Sports-related accident & $20(16.67)$ \\
Others & $20(16.67)$ \\
Total & 120 \\
\hline
\end{tabular}

Values are presented as number (\%) or mean \pm SD. ing or falling objects and injuries at work (Table 1).

In the 15D-HRQoL evaluation conducted the day after surgery, excretion $(0.13 \pm 0.13)$ was the item with the lowest score, while vision and hearing received scores of 1 , corresponding to full function (Fig. 1). Low scores were also recorded for discomfort, breathing, sleeping, distress, and speech (Table 2). At the 3-month follow-up, the distress item had the lowest score, at $0.47 \pm 0.24$ points, followed by breathing $(0.62 \pm 0.26)$, sleeping $(0.63 \pm 0.23)$, and discomfort (0.74 \pm 0.19$)$ points (Fig. 2).

Upon comparing the mean 15D-HRQoL values recorded on the day after surgery and at the 3-month follow-up, highly statistically significant $(p<0.001)$ improvements were found for breathing, sleeping, speech, excretion, and discomfort (Fig. 3). Statistically significant changes, albeit with higher $p$-values, were also found for distress $(0.10 \pm 0.18, p=0.009)$ and usual activities $(0.06 \pm 0.16, p=0.048)$ (Table 2$)$.

Regarding nasal symptoms, at 3 months after surgery, there

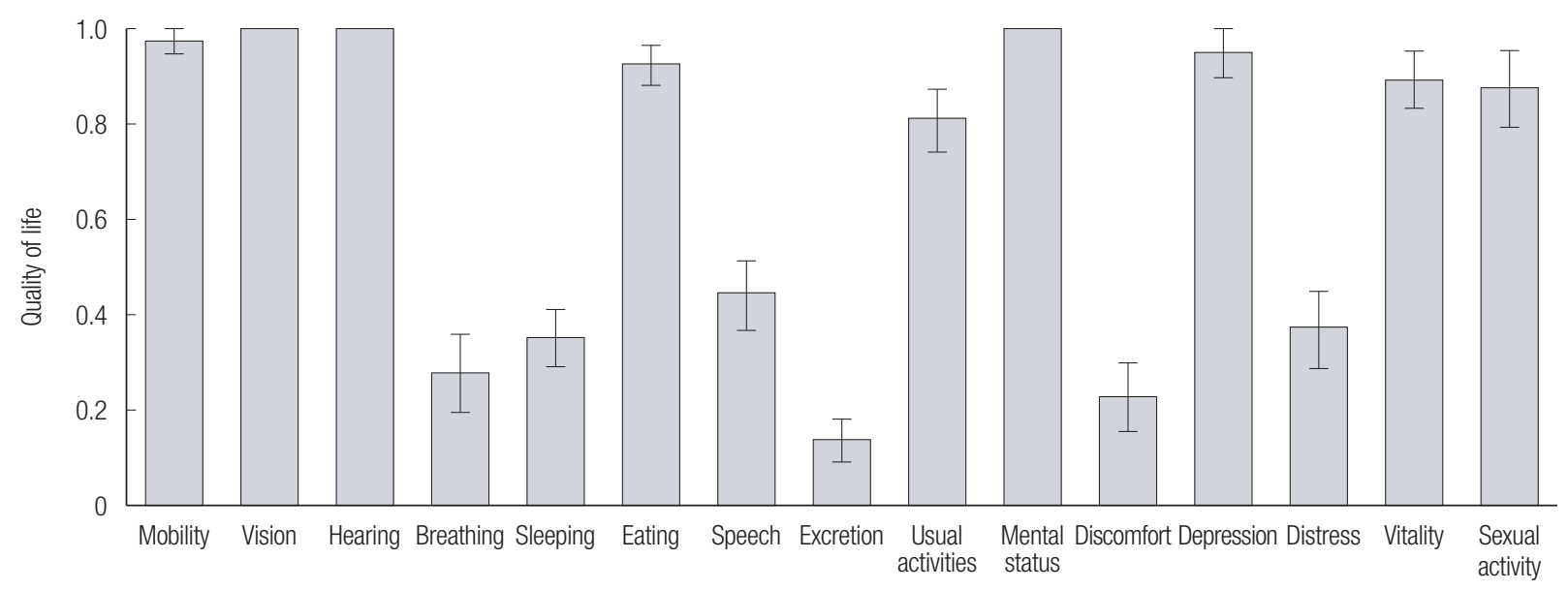

Fig. 1. Immediate postoperative (1 day) quality of life scores of patients who underwent closed reduction for a nasal bone fracture (means with $95 \%$ confidence intervals).

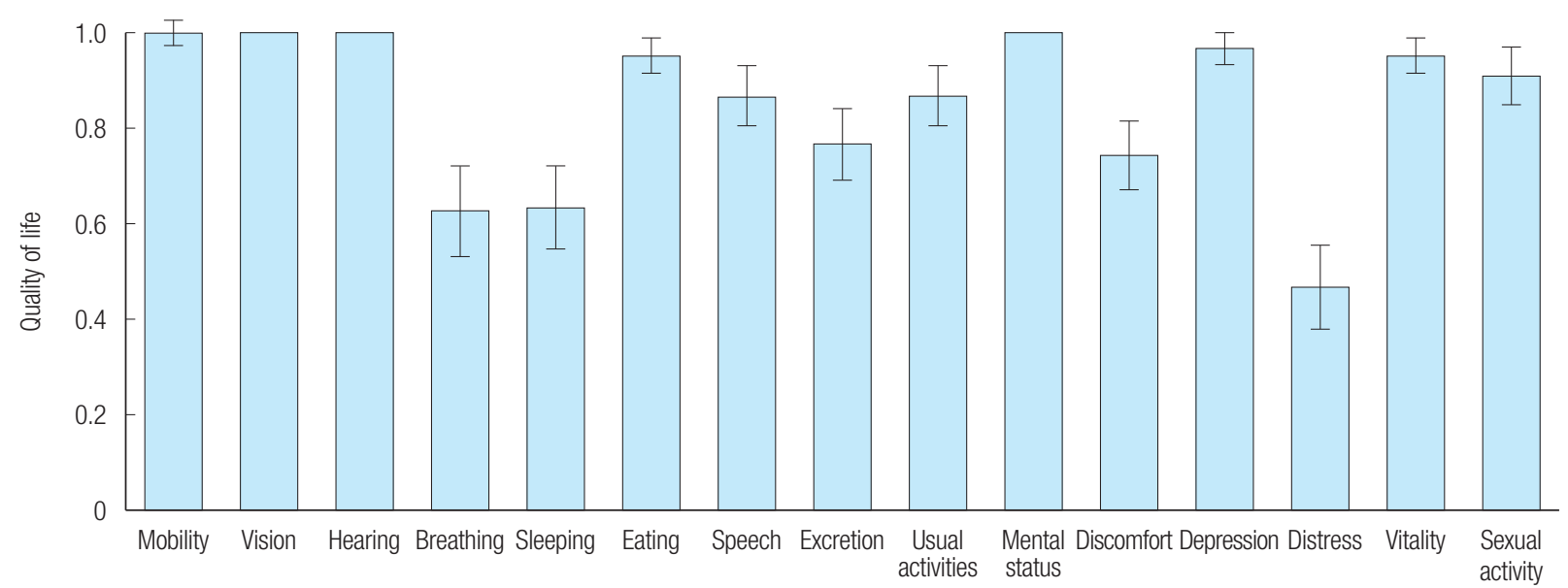

Fig. 2. Three-month follow-up quality of life scores of patients who underwent closed reduction for a nasal bone fracture (means with $95 \%$ confidence intervals). 


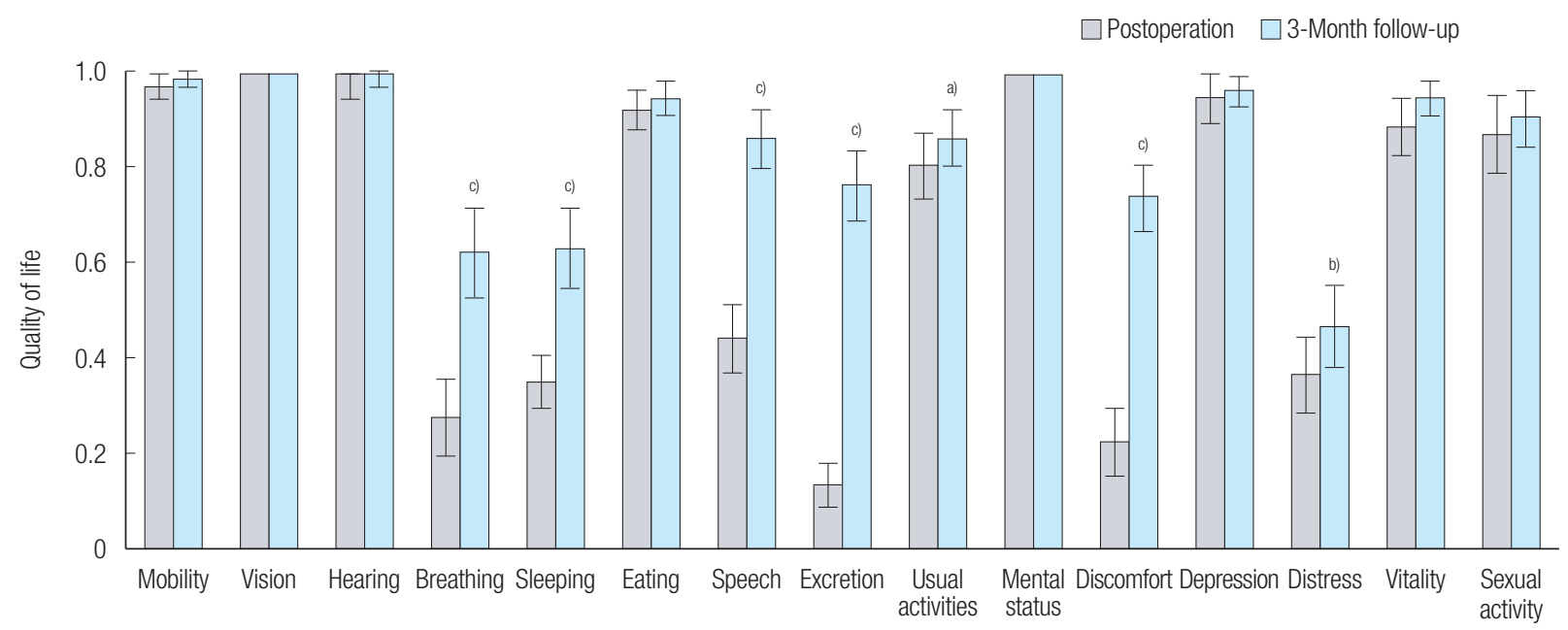

Fig. 3. Comparisons of postoperative and 3-month follow-up quality of life scores of patients who underwent closed reduction for a nasal bone fracture (means with $95 \%$ confidence intervals). ${ }^{\text {a) }} p<0.05,{ }^{\text {b) }} p<0.01,{ }^{c)} p<0.001$.

Table 2. Quality of life scores of patients who underwent closed reduction for a nasal bone fracture postoperatively and at a 3-month follow-up

\begin{tabular}{|c|c|c|c|c|}
\hline \multirow[b]{2}{*}{ Variable } & \multicolumn{3}{|c|}{ Quality of life } & \multirow[b]{2}{*}{$p$-value } \\
\hline & $\begin{array}{l}\text { Postoperative } \\
\text { (1 day) }\end{array}$ & $\begin{array}{l}\text { 3-Month } \\
\text { follow-up }\end{array}$ & $\begin{array}{l}1 \text { Day to } \\
3 \text { months }\end{array}$ & \\
\hline Mobility & $0.97 \pm 0.08$ & $0.99 \pm 0.05$ & $0.02 \pm 0.06$ & 0.346 \\
\hline Vision & 1 & 1 & 0 & - \\
\hline Hearing & 1 & 1 & 0 & - \\
\hline Breathing & $0.28 \pm 0.23$ & $0.62 \pm 0.26$ & $0.35 \pm 0.29$ & $<0.001^{\text {a) }}$ \\
\hline Sleeping & $0.35 \pm 0.16$ & $0.63 \pm 0.23$ & $0.28 \pm 0.25$ & $<0.001^{\text {a) }}$ \\
\hline Eating & $0.92 \pm 0.12$ & $0.95 \pm 0.10$ & $0.02 \pm 0.08$ & 0.149 \\
\hline Speech & $0.44 \pm 0.20$ & $0.87 \pm 0.17$ & $0.42 \pm 0.28$ & $<0.001^{\text {a) }}$ \\
\hline Excretion & $0.13 \pm 0.13$ & $0.77 \pm 0.21$ & $0.63 \pm 0.28$ & $<0.001^{\text {a) }}$ \\
\hline Usual activities & $0.81 \pm 0.19$ & $0.87 \pm 0.17$ & $0.06 \pm 0.16$ & $0.048^{\text {a) }}$ \\
\hline Mental status & 1 & 1 & 0 & \\
\hline Discomfort & $0.22 \pm 0.20$ & $0.74 \pm 0.19$ & $0.52 \pm 0.28$ & $<0.001^{\text {a) }}$ \\
\hline Depression & $0.95 \pm 0.14$ & $0.97 \pm 0.09$ & $0.02 \pm 0.06$ & 0.346 \\
\hline Distress & $0.37 \pm 0.22$ & $0.47 \pm 0.24$ & $0.10 \pm 0.18$ & $0.009^{\mathrm{a})}$ \\
\hline Vitality & $0.89 \pm 0.17$ & $0.95 \pm 0.10$ & $0.06 \pm 0.14$ & 0.053 \\
\hline Sexual activity & $0.88 \pm 0.23$ & $0.91 \pm 0.17$ & $0.03 \pm 0.09$ & 0.072 \\
\hline
\end{tabular}

Values are presented as mean \pm SD.

${ }^{a} p$-values were determined by the Wilcoxon signed-rank test, statistically significant, $p<0.05$.

were 31 patients (25.83\%) with nose obstruction, 25 (20.83\%) with snoring, $12(10.00 \%)$ with pain, 11 (9.17\%) with nasal secretions, and 29 (24.17\%) with aesthetic dissatisfaction (Table 3 ).

\section{DISCUSSION}

Closed reduction is the standard surgical method for nasal bone fractures [1-4], which are the most common fracture of the facial bones. Kaukola et al. [7] evaluated the quality of life of
Table 3. Persistent nose-related symptoms at a 3-month follow-up after surgery

\begin{tabular}{lc}
\hline Symptoms & $\begin{array}{c}\text { No. of patients with symptoms after } \\
3 \text { months (\%) }\end{array}$ \\
\hline Nose obstruction & $31(25.83)$ \\
Snoring & $25(20.83)$ \\
Pain & $12(10.00)$ \\
Nasal excretion & $11(9.17)$ \\
Aesthetic dissatisfaction & $29(24.17)$ \\
\hline
\end{tabular}

patients undergoing reduction due to zygomatic fractures and investigated the symptoms of patients who continued to complain of discomfort even months after surgery. Instead, this study dealt with nasal bone fractures, which are more common, and analyzed data from a questionnaire administered to patients the day after surgery and 3 months after surgery.

In this study, the HRQoL dimensions of excretion, discomfort, breathing, sleeping, distress, and speech had low scores the day after surgery. The low scores for excretion may be explained by excretory dysfunction resulting from general anesthesia. Furthermore, nasal packing on both sides causes discomfort, which may have resulted in difficulties in breathing and speech due to the need to breathe through the mouth. Regarding the low scores for sleeping, interviews with patients who had difficulty sleeping demonstrated that the most common sleeping problem was insomnia caused by breathing difficulties resulting from nasal packing, followed by pain.

The low scores for distress may have reflected both stress due to surgery itself and stress associated with one's cosmetic appearance. Among the six impaired dimensions of HRQoL on the day after surgery, breathing, sleeping, speech, excretion, and discomfort had significantly improved by 3 months later. The improvement in excretion scores may be explained by recovery 
from general anesthesia, and it is likely that resolution of the inconvenience posed by nasal packing accounted for the improvements in breathing, sleeping, speech, and discomfort.

At 3 months after surgery, despite significant improvements, distress, breathing, and sleeping continued to have low scores, and patients still reported difficulties in daily life. The low scores for these items and the results regarding additional nasal symptoms at 3 months after surgery imply that the possibility of breathing and sleeping difficulties being induced by nose obstruction and snoring cannot be excluded. Similarly, it cannot be ruled out that distress resulting from pain and aesthetic dissatisfaction may have contributed to low scores. In a study on long-term (after 6 months) postoperative complications by Choi et al. [2], 22.95\% of patients who received reduction after nasal bone fracture complained of functional complications regardless of fracture type, with $11.47 \%$ of patients reporting nasal obstruction, $4.92 \%$ reporting rhinorrhea, 3.28\% reporting pain, and 3.28\% reporting nasal bleeding. In addition, $22.95 \%$ of the patients had aesthetic complications, including a deviated nose in $19.67 \%$ of patients, hump nose in $1.64 \%$, and saddle nose in $1.64 \%$. These functional and aesthetic complications can contribute to reductions in quality of life.

However, the questionnaire survey conducted in this study did not allow a comparison of preoperative and postoperative results, because no data were available from before trauma. Another limitation is the lack of longer-term postoperative data. Only 3 months of follow-up cannot be considered to indicate definitive outcomes. Therefore, it is unclear whether symptoms that existed before surgery may have persisted during longterm follow-up. Additionally, the type of fracture and the surgical technique used may also affect quality of life, especially in patients with septal fractures.

In summary, HRQoL improved from the day after the closed reduction of a nasal fracture to 3 months postoperatively, but distress, breathing, and sleeping continued to cause some inconveniences, which affected HRQoL. Postoperative nasal obstruction, snoring, pain, and aesthetic dissatisfaction cannot be excluded as causes of diminished quality of life. Therefore, reduction should be performed carefully, with due consideration of septal deviation, irregularity, and asymmetry during surgery. In addition, attention should be paid to pain control after surgery as a way to improve patients' quality of life.

\section{NOTES}

\section{Conflict of interest}

No potential conflict of interest relevant to this article was reported.

\section{Ethical approval}

The study was approved by the Institutional Review Board of Gyeongsang National University Hospital (IRB No. 2020-08013-002) and performed in accordance with the principles of the Declaration of Helsinki. Written informed consents were obtained.

\section{ORCID}

Young Ji Park https://orcid.org/0000-0001-8416-7833

Gi Cheol Do https://orcid.org/0000-0002-8869-7409

Gyu Hyeon Kwon https://orcid.org/0000-0002-8377-4698

Woo Sang Ryu

Kyung Suk Lee

https://orcid.org/0000-0002-7472-3813

https://orcid.org/0000-0001-7731-1761

Nam Gyun Kim

\section{REFERENCES}

1. Rhee SC, Kim YK, Cha JH, Kang SR, Park HS. Septal fracture in simple nasal bone fracture. Plast Reconstr Surg 2004;113:4552.

2. Choi MH, Cheon JS, Son KM, Choi WY. Long-term postoperative satisfaction and complications in nasal bone fracture patients according to fracture type, site, and severity. Arch Craniofac Surg 2020;21:7-14.

3. Kang CM, Han DG. Objective outcomes of closed reduction according to the type of nasal bone fracture. Arch Craniofac Surg 2017;18:30-6.

4. Kim KS, Lee HG, Shin JH, Hwang JH, Lee SY. Trend analysis of nasal bone fracture. Arch Craniofac Surg 2018;19:270-4.

5. Muraoka M, Nakai Y. Twenty years of statistics and observation of facial bone fracture. Acta Otolaryngol Suppl 1998;538:261-5.

6. Park YJ, Ryu WS, Kwon GH, Lee KS. The clinical usefulness of closed reduction of nasal bone using only a periosteal elevator with a rubber band. Arch Craniofac Surg 2019;20:284-8.

7. Kaukola L, Snall J, Roine R, Sintonen H, Thoren H. Health-related quality of life of patients with zygomatic fracture. Med Oral Patol Oral Cir Bucal 2017;22:e636-42.

8. Sintonen $\mathrm{H}$. The $15 \mathrm{D}$ instrument of health-related quality of life: properties and applications. Ann Med 2001;33:328-36.

9. Haapaniemi TH, Sotaniemi KA, Sintonen H, Taimela E. The generic 15D instrument is valid and feasible for measuring health related quality of life in Parkinson's disease. J Neurol Neurosurg Psychiatry 2004;75:976-83. 